\title{
ON CERTAIN CONVEX SETS IN THE SPACE OF LOCALLY SCHLICHT FUNCTIONS
}

\author{
BY
}

\section{Y. J. KIM AND E. P. MERKES}

\begin{abstract}
Let $H=H(*,[+])$ denote the real linear space of locally schlicht normalized functions in $|z|<1$ as defined by Hornich. Let $K$ and $C$ respectively be the classes of convex functions and the close-to-convex functions. If $M \subset H$ there is a closed nonempty convex set in the $\alpha \beta$-plane such that for $(a, \beta)$ in this set $\alpha^{*} f[+] \beta_{g}^{*} \in C$ (in $K$ ) whenever $f, g \in M$. This planar convex set is explicitly given when $M$ is the class $K$, the class $C$, and for other classes. Some consequences of these results are that $K$ and $C$ are convex sets in $H$ and that the extreme points of $C$ are not in $K$.
\end{abstract}

1. Introduction. Let $H$ denote the class of locally schlicht analytic functions $f$ in the open unit disk $E$, normalized by the conditions $f(0)=0, f^{\prime}(0)=1$. Hornich [3] provided a linear space structure to $H$ with the operations

$$
f[+] g=\int_{0}^{z} f^{\prime}(t) g^{\prime}(t) d t, \quad \alpha^{*} f=\int_{0}^{z}\left(f^{\prime}(t)\right)^{a} d t,
$$

where $f, g \in H$ and $a$ is a real number.

This paper is primarily concerned with two convex subsets of $H$, namely, the convex schlicht functions $K$ and the close-to-convex functions $C$ [4]. More specifically, the main results are summarized in the following two theorems.

Theorem A. Let $f, g \in K$. Define, for real $a$ and $\beta$,

$$
G_{a, \beta}(z)=a^{*} f[+] \beta^{*} g=\int_{0}^{z}\left(f^{\prime}(t)\right)^{a}\left(g^{\prime}(t)\right)^{\beta} d t .
$$

Then (i) $G_{a, \beta} \in K$ if $a \geq 0, \beta \geq 0, a+\beta \leq 1$. (ii) $G_{a, \beta} \in C$ if $-1 / 2 \leq \alpha, \beta \leq$ $3 / 2,-1 / 2 \leq \alpha+\beta \leq 3 / 2$. In each case, the result is sharp.

Sharpness here means that for each pair of real numbers $\alpha, \beta$, not restricted as in the theorem, there exist functions $f, g \in K$ such that the corresponding $G_{\alpha \beta}$ is not in the stated subclass of $H$.

Theorem B. Let $f, g \in C$. Then $G_{\alpha, \beta} \in C$ if $-1 / 3 \leq \alpha+\beta \leq 1, a-3 \beta \leq 1$, $\beta-3 \alpha \leq 1$. This result is sharp.

Received by the editors June 29, 1973 and, in revised form, September 17, 1973.

AMS (MOS) subject classifications (1970). Primary $30 \wedge 32$.

Key words and phrases. Univalent, convex close-to-convex, extreme point. 
In particular these theorems prove $K$ and $C$ are convex sets in $H$. Furthermore, a line segment joining two functions in $K$ can always be extended from each end point into the convex set $C$. In particular, this implies that no extreme point of the convex set $C$ in $H$ is a convex function.

A number of authors ([5], [6], [7]) have considered the special case where only one function appears in the integrand (1). In fact, Theorem 1 and Theorem 2 reduce to theorems of Merkes and Wright [5] when $\beta=0$. The one-dimensional results concern radial lines in $H$ joining a point of $K$ or $C$ with the origin, $f(z) \equiv z$, in $H$. In addition to consideration of functions $G_{a, 0}$ earlier papers ([2], [G]) also discuss the close-to-convexity of integrals of the form

$$
g_{\alpha}(z)=\int_{0}^{z}(f(t) / t)^{\alpha} d t
$$

where $f$ is in a given subclass of $H$. This suggests a study of functions $g \in H$ such that $g \neq 0$ in $0<|z|<1$. Some special cases of this problem are discussed in $\$ 5$ of this paper.

2. Some lemmas. If $p(z)=1+a_{1} z+a_{2} z^{2}+\cdots$ in $E$ and if $\operatorname{Re} p(z)>0, z \in E$, then, by the mean value theorem of harmonic functions, we have

$$
0 \leq \int_{\theta_{1}}^{\theta_{2}} \operatorname{Re}\left\{p\left(r e^{i \theta}\right)\right\} d \theta \leq \int_{0}^{2 \pi} \operatorname{Re}\left\{p\left(r e^{i \theta}\right)\right\} d \theta=2 \pi
$$

for $0 \leq \theta_{1}<\theta_{2} \leq 2 \pi$ and for all $r, 0 \leq r<1$.

Lemma 1. If $f \in K$, then for $0 \leq r<1,0 \leq \theta_{1}<\theta_{2} \leq 2 \pi$ we have

$$
\frac{\theta_{2}-\theta_{1}}{2} \leq \int_{\theta_{1}}^{\theta_{2}} \operatorname{Re}\left\{\frac{r e^{i \theta_{f}\left(r e^{i \theta}\right)}}{f\left(r e^{i \theta}\right)}\right\} d \theta \leq \pi+\frac{\theta_{2}-\theta_{1}}{2},
$$

and

$$
0 \leq \int_{\theta_{1}}^{\theta_{2}} \operatorname{Re}\left\{1+\frac{r e^{i \theta} f^{\prime \prime}\left(r e^{i \theta}\right)}{f^{\prime}\left(r e^{i \theta}\right)}\right\} d \theta \leq 2 \pi .
$$

Proof. The inequalities (3) follow from (2) and the fact that $\operatorname{Re}\left\{z f^{\prime} / f\right\}>1 / 2$ for $f \in K$. The analytic definition of the class $K$ and (2) imply (4).

Lemma 2. If $f \in C$, then

$$
-\pi+\frac{\theta_{2}-\theta_{1}}{2} \leq \int_{\theta_{1}}^{\theta_{2}} \operatorname{Re}\left\{\frac{r e^{i \theta} f^{\prime}\left(r e^{i \theta}\right)}{f\left(r e^{i \theta}\right)}\right\} d \theta \leq 2 \pi+\frac{\theta_{2}-\theta_{1}}{2}
$$

and

$$
-\pi \leq \int_{\theta_{1}}^{\theta_{2}} \operatorname{Re}\left\{1+\frac{r e^{i \theta_{f}^{\prime \prime}\left(r e^{i \theta}\right)}}{f^{\prime}\left(r e^{i \theta}\right)}\right\} d \theta \leq 3 \pi
$$

where $0 \leq \theta_{1}<\theta_{2} \leq 2 \pi$ and $0 \leq r<1$. 
Proof. For $f \in C$ there is a function $\phi$ and a real number $\beta,|\beta|<\pi / 2$, such that $e^{-i \beta} \phi \in K$ and $\operatorname{Re}\left\{f^{\prime}(z) / \phi^{\prime}(z)\right\}>0, z \in E$. The last condition implies $\operatorname{Re}\{f(z) / \phi(z)\}>0, z \in E$, as well [5]. It follows that

$$
\left|\arg \left[\frac{f\left(r e^{i \theta}\right)}{r e^{i \theta}}\right]-\arg \left[\frac{\phi\left(r e^{i \theta}\right)}{r e^{i \theta}}\right]\right|<\frac{\pi}{2} \text {. }
$$

Now this implies

$$
\begin{aligned}
\left|\int_{\theta_{1}}^{\theta_{2}} \operatorname{Re}\left\{\frac{r e^{i \theta} f^{\prime}\left(r e^{i \theta}\right)}{f\left(r e^{i \theta}\right)}\right\} d \theta-\int_{\theta_{1}}^{\theta_{2}} \operatorname{Re}\left\{\frac{r e^{i \theta} \phi^{\prime}\left(r e^{i \theta}\right)}{\phi\left(r e^{i \theta}\right)}\right\} d \theta\right| \\
\quad=\left|\arg \frac{f\left(r e^{i \theta_{2}}\right)}{r e^{i \theta_{2}}}-\arg \frac{\phi\left(r e^{i \theta_{2}}\right)}{r e^{i \theta_{2}}}-\arg \frac{f\left(r e^{i \theta_{1}}\right)}{r e^{i \theta_{1}}}+\arg \frac{\phi\left(r e^{i \theta_{1}}\right)}{r e^{i \theta_{1}}}\right| \\
\leq \pi / 2+\pi / 2=\pi .
\end{aligned}
$$

Since $e^{-i \beta} \phi \in K$ the last inequality and (3) imply (5). The left-hand side of (6) is Kaplan's characterization of close-to-convex functions [4]. The right-hand inequality in (6) follows from (4) and the fact that

$$
\left|\arg f^{\prime}\left(r e^{i \theta}\right)-\arg \phi^{\prime}\left(r e^{i \theta}\right)\right|<\pi / 2 \text {. }
$$

The next lemma provides a class of close-to-convex functions that serve as extremal functions for the sharpness arguments in this paper.

Lemma 3. For real $a$, the function $b_{a}(z)=\int_{0}^{z}(1+t)^{a} d t$ is not univalent in $E$ if $a \notin[-3,1]$. If $-3 \leq a \leq 1$, then $b_{a}$ is close-to-convex in $E . b_{a}$ is convex if and only if $-2 \leq a \leq 0$.

Proof. $b_{a}(z)=\left[(1+z)^{a+1}-1\right] /(1+\alpha)$ provided $a \neq-1$. When $a=-1$, this function is $\log (1+z)$ which is univalent and convex in $E$. If $a \neq-1, b_{a}$ is univalent in $E$ if and only if $(1+z)^{a+1}$ is univalent. The latter is the case if and only if $-3 \leq \alpha \leq 1$ [7]. For $-3 \leq \alpha \leq-1$, furthermore, let $\phi(z)=z /(1+z)$ and we have

$$
\operatorname{Re} \frac{b_{a}^{\prime}(z)}{\phi^{\prime}(z)}=\operatorname{Re}(1+z)^{a+2} \geq 0, z \in E .
$$

For $-1<\alpha \leq 1$, let $\phi=z$ and $\operatorname{Re}\left\{b_{\alpha}^{\prime}(z) / \phi^{\prime}(z)\right\}=\operatorname{Re}(1+z)^{a}>0, z \in E$. This proves $b_{a}$ is close-to-convex for $-3 \leq a \leq 1$. The convexity follows the fact, for real $a$,

$$
\operatorname{Re}\left\{\frac{z b_{a}^{\prime \prime}(z)}{b_{a}^{\prime}(z)}+1\right\}=\operatorname{Re} \frac{1+(1+a) z}{1+z} \geq 0
$$

if and only if $-2 \leq a \leq 0$. 
3. Convexity results. The first question that we consider is a proof of the convexity of the sets $K$ and $C$ in the space $H$. The result for $K$ is known [2].

Theorem 1. Let $f_{1}, f_{2}$ be in $K$ (in $C$ ) and let $0 \leq \lambda \leq 1$. Then $G_{\lambda, 1-\lambda}$ given by (1) is in $K$ (in $C$ ).

Proof. If $f_{1}, f_{2}$ are in $K$, then from (1) we obtain

$$
\operatorname{Re}\left\{\frac{z G_{\lambda, 1-\lambda}^{\prime \prime}}{G_{\lambda, 1-\lambda}^{\prime}}+1\right\}=\lambda \operatorname{Re}\left\{\frac{z f_{1}^{\prime \prime}}{f_{1}^{\prime}}+1\right\}+(1-\lambda) \operatorname{Re}\left\{\frac{z f_{2}^{\prime \prime}}{f_{2}^{\prime}}+1\right\}
$$

which is nonnegative since $f_{1}$ and $f_{2}$ are in $K$. It follows that $G_{\lambda, 1-\lambda} \in K$. Now assume $f_{j} \in C$. Then there exist functions $\phi_{j} \in K$ and real numbers $\beta_{j},\left|\beta_{j}\right|<$ $\pi / 2$, such that

$$
\operatorname{Re}\left\{e^{i \beta_{j}} f_{j}^{\prime}(z) / \phi_{j}^{\prime}(z)\right\}>0, \quad z \in E \quad(j=1,2)
$$

Define

$$
\phi_{\lambda, 1-\lambda}(z)=\int_{0}^{z}\left(\phi_{1}^{\prime}(t)\right)^{\lambda}\left(\phi_{2}^{\prime}(t)\right)^{1-\lambda} d t
$$

and, by the first part of this theorem, $\phi_{\lambda, 1-\lambda} \in K$. Furthermore,

$$
e^{i \beta} \frac{G_{\lambda, 1-\lambda}^{\prime}}{\phi_{\lambda, 1-\lambda}^{\prime}}=\left(e^{i \beta_{1}} \frac{f_{1}^{\prime}}{\phi_{1}^{\prime}}\right)^{\lambda}\left(e^{i \beta_{2}} \frac{f_{2}^{\prime}}{\phi_{2}^{\prime}}\right)^{1-\lambda}
$$

where $\beta=\lambda \beta_{1}+(1-\lambda) \beta_{2}$. In view of (7), it follows that the real part of the lefthand side of (8) is nonnegative and, hence, that $G_{\lambda, 1-\lambda}$ is close-to-convex [4].

Let $f_{1}$ and $f_{2}$ be two functions in $H$ and assume the corresponding $G_{a, \beta}$ function ( 1 ) is in $C$ (in $K$ ) for $\alpha=\alpha_{1}, \beta=\beta_{1}$ and for $\alpha=\alpha_{2}, \beta=\beta_{2}$. Then $G_{a, \beta}$ is in $C$ (in $K$ ) for all points in the $\alpha \beta$-plane on the line segment joining $\left(\alpha_{1}, \beta_{1}\right)$ and $\left(\alpha_{2}, \beta_{2}\right)$. Indeed, the function

$$
\begin{aligned}
H_{\lambda}(z) & =\int_{0}^{z}\left(G_{a_{1}, \beta_{1}}^{\prime}(t)\right)^{\lambda}\left(G_{a_{2}, \beta_{2}}^{\prime}(t)\right)^{1-\lambda} d t \\
& =\int_{0}^{z}\left(f_{1}^{\prime}(t)\right)^{\lambda a_{1}+(1-\lambda) a_{2}}\left(f_{2}^{\prime}(t)\right)^{\lambda \beta_{1}+(1-\lambda) \beta_{2}} d t
\end{aligned}
$$

is in $C$ (in $K$ ) for $0 \leq \lambda \leq 1$ by Theorem 1. It follows that for each pair $f_{1}, f_{2} \in H$ there is a convex set in the $\alpha \beta$-plane containing $(0,0)$ and such that the corresponding $G_{a, \beta}$ function of the pair is in $C$ (in $K$ ). Furthermore, this set is closed in the plane since $C$ (or $K$ ) is a compact subclass of $H$ in the topology of uniform convergence. These remarks assist in the proof of the following useful result.

Lemma 4. Let $M$ be a nonempty subclass of $H$. Then the set of points in the 
$a \beta$-plane such that $G_{a, \beta}$ is in $C$ (in $K$ ) for each pair $f_{1}, f_{2}$ in $M$ is nonempty, closed, and convex.

Proof. For each pair $f, g \in M$ there is a maximal closed convex set in the $\alpha \beta$ plane such that the functions $G_{a, \beta}$ in (1) are in $C$ (in $K$ ). The intersection of these sets, taken over all pairs $f, g \in M$, is a closed, convex set containing the origin.

4. Proofs of the principal theorems. We begin with a proof of part (i) of Theorem A. Let $f_{1}, f_{2}$ be in $K$. Then from (1)

$$
\begin{aligned}
R_{a, \beta}(z) & \equiv \operatorname{Re}\left\{1+\frac{z G_{a, \beta}^{\prime \prime}(z)}{G_{a, \beta}^{\prime}(z)}\right\} \\
& =(1-\alpha-\beta)+\alpha \operatorname{Re}\left\{1+\frac{z f_{1}^{\prime \prime}(z)}{f_{1}^{\prime}(z)}\right\}+\beta \operatorname{Re}\left\{1+\frac{z f_{2}^{\prime \prime}(z)}{f_{2}^{\prime}(z)}\right\}
\end{aligned}
$$

which is clearly nonnegative if $a \geq 0, \beta \geq 0$, and $\alpha+\beta \leq 1$. For the sharpness, let $f_{1}=f_{2}=z /(1+z)$. Then

$$
G_{a, \beta}(z)=\int_{0}^{z} \frac{d t}{(1+t)^{2(\alpha+\beta)}},
$$

and, by Lemma 3, this function is in $K$ if and only if $0 \leq a+\beta \leq 1$. The fact that $a \geq 0$ follows from consideration of $G_{a, \beta}$ for $f_{1}=z /(1+z)$ and $f_{2}=z$. We obtain $\beta \geq 0$ by symmetry.

To prove (ii) of Theorem $A$, we first observe that the closed convex region $-1 / 2 \leq \alpha, \beta, \alpha+\beta \leq 3 / 2$ is the convex hull of the points $(3 / 2,0),(0,3 / 2)$, $(-1 / 2,0),(0,-1 / 2),(3 / 2,-1 / 2)$, and $(-1 / 2,3 / 2)$. That the first four of these points are contained in the convex set $D$, that exists by Lemma 4 with $M=K$, is proved in [5]. For $a=3 / 2, \beta=-1 / 2$ we have from (9) that

$$
\begin{aligned}
R_{3 / 2,-1 / 2}(z) & =\frac{3}{2} \cdot \operatorname{Re}\left\{1+\frac{z f_{1}^{\prime \prime}(z)}{f_{1}^{\prime}(z)}\right\}-\frac{1}{2} \cdot \operatorname{Re}\left\{1+\frac{z f_{2}^{\prime \prime}(z)}{f_{2}^{\prime}(z)}\right\} \\
& \geq-\frac{1}{2}: \operatorname{Re}\left\{1+\frac{z f_{2}^{\prime \prime}(z)}{f_{2}^{\prime}(z)}\right\} .
\end{aligned}
$$

Since $f_{2} \in K$, this inequality and Lemma 1 imply

$$
\int_{\theta_{1}}^{\theta} R_{3 / 2,-1 / 2}\left(r e^{i \theta}\right) d \theta \geq-\frac{1}{2}(2 \pi)=-\pi
$$

from which we conclude that $G_{3 / 2,-1 / 2} \in C$ [4].

To verify the sharpness of the region in (ii) of Theorem $A$, consider the con- 
vex functions $z$ and $z /(1+z)$. With $f_{1}=z, f_{2}=z /(1+z)$ we obtain $-1 / 2 \leq \beta$ $\leq 3 / 2$ and $-1 / 2 \leq \alpha \leq 3 / 2$ follows by symmetry. The remaining restrictions on $\alpha$ and $\beta$ are established by setting $f_{1}=f_{2}=z /(1+z)$.

We turn now to the proof of Theorem $B$. The convex set in the $\alpha \beta$-plane in this theorem is simply the closed convex hull of the points $(1,0),(0,1),(-1 / 3,0)$ and $(0,-1 / 3)$. For each of these points it has been proved [5] that $G_{a, \beta} \in C$ whenever $f_{1}, f_{2} \in C$. Hence, by Lemma $4, G_{a, \beta} \in C$ for all $\alpha, \beta$ in this convex hull. The sharpness of this closed convex set remains to be established.

The function $s(z)=z(1+1 / 2 z) /(1+z)^{2}$ is close-to-convex relative to the convex function $\phi(z)=z /(1+z)$. Let $f_{1}(z)=f_{2}(z)=s(z)$ in (1). Then $G_{a, \beta}(z)=$ $\int_{0}^{z}(1+t)^{-3 a-3 \beta} d t$. By Lemma 3 , this function is univalent in $E$ if and only if $-1 / 3 \leq \alpha+\beta \leq 1$ and is close-to-convex for $\alpha$ and $\beta$ in this interval. Thus $G_{a, \beta}$ is not univalent for the given pair of functions whenever $\alpha+\beta>1$ or $\alpha+$ $\beta<-1 / 3$. If we set $f_{1}(z)=z$ and $f_{2}(z)=s(z)$ then $G_{\alpha, \beta}^{\prime}=(1+z)^{-3 \beta}$ from which we conclude $G_{a, \beta}$ is not univalent for $\beta>1$ or $\beta<-1 / 3$. The restrictions on $\alpha$ are obtained by symmetry and the sharpness of the closed region in Theorem $B$ is proved.

A curious result is obtained when $f_{1}$ is in $C$ while $f_{2}$ is restricted to $K$.

Theorem C. If $f_{1} \in C, f_{2} \in K$, then $G_{\alpha \beta} \in C$ when

$$
-1 / 3 \leq \alpha,-1 \leq 3 \alpha+2 \beta \leq 3,-3 \leq \alpha-2 \beta \leq 1 .
$$

This result is sharp.

Proof. The convex set $(10)$ is the closed convex hull of the points $(1,0)$, $(-1 / 3,0),(0,-1 / 2),(0,3 / 2)$ and $(-1 / 3,4 / 3)$. All, except the last, are known [5] to be points for which $G_{a, \beta} \in C$ whenever $f_{1} \in C$ and $f_{2} \in K$. The proof that $(-1 / 3,4 / 3)$ is also such a point parallels the proof of the analogous result for $(3 / 2,-1 / 2)$ in the justification of Theorem $A$. Sharpness of the first inequality follows by letting $f_{1}=z /(1+z)$ and $f_{2}=z$. The sharpness of the next two sets of inequalities in (10) are respectively established by setting $f_{1}(z)=z(1+1 / 2 z) /(1+z)^{2}$, $f_{1}(z)=z(1+1 / 2 z)$ and setting $f_{2}=z /(1+z)$ in each case. The conclusion is obtained from these functions and Lemma 3.

5. Related results.

Theorem 2. Let $f_{1}, f_{2}$ be in $K$. Define

$$
g_{a, \beta}(z)=\int_{0}^{z}\left(\frac{f_{1}(t)}{t}\right)^{a}\left(\frac{f_{2}(t)}{t}\right)^{\beta} d t .
$$

Then (i) $g_{a, \beta} \in K$ provided $\alpha \geq 0, \beta \geq 0$, and $\alpha+\beta \leq 2$ and (ii) $g_{a, \beta} \in C$ provided $-1 \leq \alpha \leq 3,-1 \leq \beta \leq 3$, and $-1 \leq \alpha+\beta \leq 3$. These results are sharp. 
Proof. The convexity of the set $D$ in the $\alpha \beta$-plane such that $g_{a, \beta} \in K$ (in $C$ ) for all $f_{1}, f_{2} \in K$ follows from Lemma 4 by setting $M=\left\{g \in H: z g^{\prime} \in K\right\}$ (i) is a simple consequence of this since $(0,0),(2,0),(0,2)$ are in $D$ by [5]. The closed convex region in (ii) is the closed convex hull of the points $(-1,0),(0,-1)$, $(3,0),(0,3),(3,-1),(-1,3)$. The first four are known $[5]$ to be in the set $D$ such that $g_{a, \beta} \in C$ for all $f_{1}, f_{2} \in K$. To prove $(3,-1)$ is also in $D$, we observe that

$$
\begin{aligned}
M_{3,-1}(z) & \equiv \operatorname{Re}\left\{1+\frac{z g_{3,-1}^{\prime \prime}(z)}{g_{3,-1}^{\prime}(z)}\right\} \\
& =-1+3 \operatorname{Re}\left\{\frac{z f_{1}^{\prime}(z)}{f_{1}(z)}\right\}-\operatorname{Re}\left\{\frac{z f_{2}^{\prime}(z)}{f_{2}(z)}\right\} \\
& \geq \frac{1}{2}-\operatorname{Re}\left\{\frac{z f_{2}^{\prime}(z)}{f_{2}(z)}\right\}
\end{aligned}
$$

since $\operatorname{Re}\left\{z f_{1}^{\prime} / f_{1}\right\} \geq 1 / 2$ for $f_{1} \in K$. Let $0 \leq \theta_{1}<\theta_{2} \leq 2 \pi$ and integrate $M_{3,-1}\left(r e^{i \theta}\right)$ with respect to $\theta$ from $\theta_{1}$ to $\theta_{2}$ for a fixed $r, 0 \leq r<1$. By (3) in Lemma 1 and the above inequality, we have

$$
\int_{\theta_{1}}^{\theta_{2}} M_{3,-1}\left(r e^{i \theta}\right) d \theta \geq \frac{\theta_{2}-\theta_{1}}{2}-\left(\pi+\frac{\theta_{2}-\theta_{1}}{2}\right)=-\pi \text {. }
$$

This proves $g_{3,-1} \in C$ [4]. The fact that $g_{-1,3} \in C$ follows by symmetry. An application of Lemma 4 now completes the proof of (ii). Sharpness in (i) and in (ii) follows by considering the functions (11) obtained by the pairing $z /(1+z)$ with itself or with $z$ for the choices of $f_{1}$ and $f_{2}$.

A similar argument to the proof of Theorem B can be used to prove the next result.

Theorem 3. If $f_{1}, f_{2} \in C$, then the function (11) is in $C$ if $(\alpha, \beta)$ belongs to the closed convex region $D$ given by $-1 / 2 \leq \alpha+\beta \leq 1, \alpha-2 \beta \leq 1$ and $\beta-$ $2 \alpha \leq 1$. The result is sharp.

The extremal functions for the first inequalities are

$$
f_{1}(z)=f_{2}(z)=\frac{z(1+\mu z)}{(1+z)^{2}}, \quad \mu=\cos \gamma e^{i \gamma}, \quad 0<\gamma<\pi
$$

(see [5]). For the next set of inequalities in the definition of $D$ the extremal functions are $f_{1}$ as given above and $f_{2}=z /(1+\mu z)^{2}, \mu=\cos \gamma e^{i \gamma}, 0<\gamma<\pi$.

Finally, the analog of Theorem $C$ for functions of the form (11) can be proved by the methods in this paper.

Theorem 4. If $f_{1} \in C$ and $f_{2} \in K$, then the function $g_{\alpha, \beta}$, defined by (11), be- 
longs to $C$ if $(\alpha, \beta)$ satisfies all the following inequalities $-1 / 2 \leq \alpha \leq 1,-1 \leq$ $2 a+\beta \leq 3,-1 \leq \beta-\alpha \leq 3$. The result is sharp.

Sharpness is obtained from various pairings of the functions $z, z /(1+\mu z)$ in $K$ and $z(1+\mu z) /(1+z)^{2}$ in $C$ where $\mu=\cos \gamma e^{i \gamma}, 0 \leq \gamma<\pi$.

\section{REFERENCES}

1. W. M. Causey, The close-to-convexity and univalence of an integral, Math. Z. 99 (1967), 207-212. MR 35 \#6807.

2. J. A. Cima and J. A. Pfaltzgraff, A Banach space of locally univalent functions, Michigan Math. J. 17 (1970), 321-334. MR $43 \# 3784$.

3. H. Hornich, Ein Banachraum analytischer Funktionen in Zusammenhang mit den schlichten Funktionen, Monatsh. Math. 73 (1969), 36-45. MR 39 \#4411.

4. W. Kaplan, Close-to-convex schlicht functions, Michigan Math. J. 1 (1952), 169-185. MR 14, 966.

5. E. P. Merkes and D. J. Wright, On the univalence of a certain integral, Proc. Amer. Math. Soc. 27 (1971), 97-100. MR 42 \#4720.

6. M. Nunokowa, $O_{n}$ the univalence of a certain integral, Trans. Amer. Math. Soc. 146 (1969), 439-446. MR 40 \#4436.

7. W. C. Royster, On the univalence of a certain integral, Michigan Math. J. 12 (1965), 385-387. MR $32 \# 1342$.

AIR FORCE ACADEMY, SEOUL, KOREA

DEP ARTMENT OF MATHEMATICS, UNIVERSITY OF CINCINNATI, CINCINNATI, OHIO 45221 